Mathematical Modelling and Analysis

Volume 22 Number 2, March 2017, 140-156

https://doi.org/10.3846/13926292.2017.1276983

(C) Vilnius Gediminas Technical University, 2017
Publisher: Taylor\&Francis and VGTU

http://www.tandfonline.com/TMMA

ISSN: $1392-6292$

eISSN: $1648-3510$

\title{
Harmonic Balance for Non-Periodic Hyperbolic Solutions of Nonlinear Ordinary Differential Equations
}

\section{Serge Bruno Yamgoué ${ }^{a}$, Olivier Tiokeng Lekeufack ${ }^{b}$ and Timoléon Crépin Kofanée , $^{b}$}

${ }^{a}$ Department of Physics, Higher Teachers Training College Bambili, The University of Bamenda

Po. Box 39 Bamenda, Cameroon

${ }^{b}$ Laboratoire de Mécanique, Département de Physique, Faculté de Sciences, Université de Yaoundé I

B.P. 812 Yaoundé - Cameroon

${ }^{c}$ Centre d'Excellence Africain des Technologies de l'Information et de la Communication (CETIC), Université de Yaoundé I

Yaoundé, Cameroon

E-mail(corresp.): sergebruno@yahoo.fr

E-mail: lekeufackolivier@gmail.com

E-mail: tckofane@yahoo.com

Received April 26, 2016; revised December 20, 2016; published online March 1, 2017

\begin{abstract}
In this paper, we propose a new approach for obtaining explicit analytical approximations to the homoclinic or heteroclinic solutions of a general class of strongly nonlinear ordinary differential equations describing conservative singledegree-of-freedom systems. Through a simple and explicit change of the independent variable that we introduce, these equations are transformed to others for which the original homoclinic or heteroclinic solutions are mapped into periodic solutions that satisfy some boundary conditions. Recent simplified methods of harmonic balance can then be exploited to construct highly accurate analytic approximations to these solutions. Here, we adopt the combination of Newton linearization with the harmonic balance to construct the approximates in incremental steps, thereby proposing both appropriate initial approximates and increments that together satisfy the required boundary conditions. Three examples including a septic Duffing oscillator, a controlled mechanical pendulum and a perturbed $\mathrm{KdV}$ equations are presented to illustrate the great accuracy and simplicity of the new approach.
\end{abstract}

Keywords: harmonic balance, linearization, explicit approximations, solitons, hyperbolic solutions.

AMS Subject Classification: 34A45; 34C37; 35A24; 35A35. 


\section{Introduction}

Physical, engineering, and biological sciences are continuously generating problems of either theoretical or practical interest. The necessary investigations of these problems involve models that, very often, are mathematically expressed as ordinary differential equations (ODEs). The solutions of the latter are therefore of great importance for the refinement of the designed models in view of the best understanding of the original problems or phenomena and, hence, their optimal exploitation or eventually their control. In this respect, solving ODEs constitutes an important research activity which is ever attracting a great deal of attention.

The ideal approach to this task is analytical. Yet, it appears to be very challenging owing to the fact that most of the relevant ODEs, being nonlinear, avail no general solution methodology. In other worlds, exact general solutions of nonlinear ODEs are rarely obtainable analytically in closed form. Even if a solution can be found, its expression is often too complicated to display clearly the principal features of the solution; this is particularly true of implicit solutions and of solutions which are in the form of integrals or infinite series [15]. Similarly, an exact analytical solution expressed in terms of special functions can be useless if it is to be used numerically (e.g. as the initial condition for a propagation problem) in a computing system that lacks these functions. In this context, several techniques have been developed to tackle the problems whenever some special circumstances can be met. For instance, approximate solutions can be obtained analytically using various perturbation techniques [21] for nonlinear ODEs which contain a small parameter. Problems with two or more scales of variation can be analyzed using the method of multiple scales [21] or the method of averaging [22].

Obviously, it is of interest to broaden as much as possible the applicability of these approximation schemes; especially when they are simple and operationally easy to use. For the important class of systems with oscillatory behavior, dedicated research efforts of recent decades have achieved this goal to some extend for some of the applicable perturbation methods; by enlarging the problems' parameters range and oscillations' amplitudes for which the methods remain accurate. A typical example here, among several others, is the famous method of Lindstedt-Poincaré which has been improved considerably through its combination with the linear delta expansion [3] or the technique of expansion of constant [14,26] (see also [4, pp. 14-19]). In addition to those works geared toward the approximation of periodic solutions, some others have recently broadened the application of the method of Lindstedt-Poincaré to the approximation of homoclinic and heteroclinic solutions $[7,8,9]$. Similarly, the renormalization group has recently been shown to be a simple and powerful approach for finding global approximate solutions to nonlinear perturbed partial differential equations arising in mathematical physics [16].

Toward the same goal, we aim in this paper to demonstrate the applicability of the well-known method of harmonic balance (HB) to the approximation of some types of solutions that are not its original target. Specifically, we show that non-periodic hyperbolic solutions of some classes of nonlinear ODEs, in- 
cluding homoclinic and heteroclinic solutions which represent solitary waves for propagation problems, can be successfully approximated using the method of $\mathrm{HB}$ and its varied derivatives. For such an ODE and provided that its periodic solutions can be approximated using the HB, we propose a simple classical change of the independent variable that puts the ODE in a form for which the subsequent HB obtained solutions approximate homoclinic or heteroclinic solutions rather than periodic ones.

The remaining part of this paper is organized as follows. In the next Section, the specific class of ODEs dealt with and the change of variable are successively presented. Then the method of HB is briefly reviewed with emphasis on aspects that are relevant for the homoclinic and heteroclinic solutions. We proceed with our examples in Section 3 where a septic Duffing oscillator, a controlled mechanical pendulum and a perturbed KdV equations are treated. Section 3.3 closes the paper with our conclusions and remarks.

\section{Outline of the approach}

We are concerned in this paper by systems whose states, represented by a scalar real variable $(x)$, evolve in time $(t)$ as prescribed by a second-order nonlinear ODE of the general form

$$
\ddot{x}+g(x) \dot{x}^{2}+f(x)=0 .
$$

Here, an overdot denotes differentiation with respect to $t$, and $f$ and $g$ are two real-valued functions. We restrict ourselves without loss of generality to conservative systems. If there exists a point $x^{(s)}$ and an integer $n \geq 0$ such that

$$
\frac{\mathrm{d}^{j} f}{\mathrm{~d} x^{j}}\left(x^{(s)}\right)=0, \quad 0 \leq j \leq 2 n ; \quad \frac{\mathrm{d}^{2 n+1} f}{\mathrm{~d} x^{2 n+1}}\left(x^{(s)}\right)>0,
$$

then, $x^{(s)}$ is said to be a stable equilibrium position for a system governed by Eq. (2.1). The system's behavior around such a point is oscillatory. This case has largely been investigated in recent decades with the assigned initial conditions $(x(0), \dot{x}(0))=(A, 0)$, with $A$ conveniently close to, but different from $x^{(s)}$. One of the successful methods that have been employed is that of HB. We assume in this section, once again without loss of generality, that the ideal conditions where it works genuinely well are satisfied: $f(-x)=-f(x)$ and $g(-x)=-g(x)$. Mikens refers to systems that fulfill these conditions as having odd-parity [20]. In this paper, we are interested by the case where Eq. (2.1) also admits unstable equilibrium solutions in addition to the stable ones. Similar to Eq. (2.2), these are determined by

$$
\frac{\mathrm{d}^{j} f}{\mathrm{~d} x^{j}}\left(x^{(u)}\right)=0, \quad 0 \leq j \leq 2 m ; \quad \frac{\mathrm{d}^{2 m+1} f}{\mathrm{~d} x^{2 m+1}}\left(x^{(u)}\right)<0,
$$

where $m \geq 0$ is an integer. In particular, for $m=0$ as assumed onward, these fixed points are hyperbolic. When the two conditions in Eq. (2.2) and Eq. (2.3) are simultaneously satisfied, there exists a non-periodic solution, $x_{h}(t)$, that is 
both forward and backward asymptotic to an unstable point:

$$
\lim _{t \rightarrow-\infty} x_{h}(t)=x_{-}^{(u)}, \quad \lim _{t \rightarrow+\infty} x_{h}(t)=x_{+}^{(u)}, \quad \lim _{t \rightarrow \pm \infty} \dot{x}_{h}(t)=0 .
$$

The corresponding trajectory is qualified as homoclinic when $x_{-}^{(u)}=x_{+}^{(u)} \equiv$ $x_{ \pm}^{(u)}$, or heteroclinic when $x_{-}^{(u)} \neq x_{+}^{(u)}$. In comparison to the effort devoted to the approximation of periodic solutions of Eq. (2.1), very few available works deal with these special orbits $[7,8,9,11,12]$. To improve on this situation we introduce a simple change of independent variable from $t$ to $\tau$ according to

$$
t=\frac{\ln \left(\tan \left(\frac{\tau}{2}+\frac{\pi}{4}\right)\right)}{\sqrt{\Omega}},
$$

where $\Omega$ is a positive quantity that must be determined as part of the approximation. Using the prime to denote a differentiation with respect to $\tau$, this change of variable transforms the differential equation Eq. (2.1) as

$$
\frac{\Omega}{2}\left[(1+\cos (2 \tau))\left(x^{\prime \prime}+g(x)\left(x^{\prime}\right)^{2}\right)-x^{\prime} \sin (2 \tau)\right]+f(x)=0 .
$$

It also transforms the first two boundary conditions in Eq. (2.4) as

$$
\lim _{\tau \rightarrow-\frac{\pi}{2}} x_{h}(\tau)=x_{-}^{(u)}, \quad \lim _{\tau \rightarrow+\frac{\pi}{2}} x_{h}(\tau)=x_{+}^{(u)}
$$

while the third one is automatically satisfied since $\dot{x}=\sqrt{\Omega} \cos (\tau) x^{\prime}$; assuming that $x^{\prime}$ remains finite.

Now, if the HB can be applied to Eq. (2.1), which requires that one be able to compute the Fourier series of $f(x(t))$ and $g(x(t))$ for a given truncation of the periodic Fourier expansion of $x(t)$, then it should equally be applicable to Eq. (2.6). This is the essence of the approach that we propose herein for the analytical construction of explicit approximations to the hyperbolic homoclinic or heteroclinic of ODEs. For the specific class of problems governed by Eq. (2.1), we suggest to initiate the approximation of homoclinic solution with

$$
x_{0}(\tau)=x_{ \pm}^{(u)}+A \cos (\tau) .
$$

For heteroclinic solution, an appropriate starting ansatz is given by

$$
x_{0}(\tau)=\frac{x_{+}^{(u)}+x_{-}^{(u)}}{2}+\frac{x_{+}^{(u)}-x_{-}^{(u)}}{2} \sin (\tau) .
$$

Motivated by the case of ODEs that describe traveling waves of PDEs, and for simplicity, we shall assume that the "amplitude" $A$ appearing in Eq. (2.8) above is given. For ODEs describing mechanical systems, it can be determined from the conservation of total mechanical energy between $x_{ \pm}^{(u)}$ and the turning point of the orbit. It may be necessary to resort to a numerical procedure to this end; just as for finding $x_{-}^{(u)}, x_{+}^{(u)}$ or $x_{ \pm}^{(u)}$. Then, a simple application of HB 
to Eq. (2.1) using either of Eq. (2.8) or Eq. (2.9) enables to find $\Omega$ and thus a first approximation to the problems.

It is noteworthy to observe that all the advantages [19] of the HB can be exploited within our proposed technique. For instance, the rational harmonic balance $[5,28]$ or the combination of HB with Newton-like linearization (HBwL) $[17,18,23,29]$ can be used for the construction of improved approximate solutions. The important point to lay emphasis on here is that each approximate solution or ansatz should satisfy the boundary conditions in Eq. (2.7), similar to the fact that prescribed initial conditions must be satisfied when applying the ordinary HB. For Eq. (2.1), we propose following the HBwL that corrections to an approximate solution be computed incrementally using the form $[29]$

$$
x_{j+1}(\tau)=x_{j}(\tau)+\sum_{i=1}^{j+1} C_{j+1,2 i+1}(\cos (\tau)-\cos ((2 i+1) \tau)),
$$

or

$$
x_{j+1}(\tau)=x_{j}(\tau)+\sum_{i=1}^{j+1} S_{j+1,2 i+1}\left(\sin (\tau)-(-1)^{i} \sin ((2 i+1) \tau)\right)
$$

for homoclinic or heteroclinic orbit, respectively. Notice that for consistency, equations Eq. (2.10) and Eq. (2.11) are designed so as to satisfy $x_{j+1}(0)=$ $x_{0}(0)$. Taking advantage of the fact that Eq. (2.6) is linear in $\Omega$, we choose to recompute it anew at each stage from the equation corresponding to the coefficient of $\cos (\tau)$ or $\sin (\tau)$ in the set of equations obtained as ordinarily in the HB. The remaining equations are next linearized respect with to, and then easily solved for

$$
\left\{C_{j+1,2 i+1}, \quad i=1,2, \cdots j+1\right\}
$$

or

$$
\left\{S_{j+1,2 i+1}, \quad i=1,2, \cdots j+1\right\}
$$

as appropriate. Once the solution is obtained in terms of $\tau$, one first converts any higher order harmonic of the form $\cos (k \tau)$ or $\sin (k \tau)$ contained in it into a polynomial in $\cos (\tau)$ and $\sin (\tau)$ using the expansions $[13$, p. 33]

$$
\begin{aligned}
\cos (k \tau)= & 2^{k-1} \cos ^{k}(\tau)-\frac{k}{1} 2^{k-3} \cos ^{k-2}(\tau)+\frac{k}{2}\left(\begin{array}{c}
k-3 \\
1
\end{array}\right) 2^{k-5} \cos ^{k-4}(\tau) \\
& -\frac{k}{3}\left(\begin{array}{c}
k-4 \\
2
\end{array}\right) 2^{k-7} \cos ^{k-6}(\tau)+\cdots, \\
\sin (k \tau)= & \sin (\tau)\left[2^{k-1} \cos ^{k-1}(\tau)-\left(\begin{array}{c}
k-2 \\
1
\end{array}\right) 2^{k-3} \cos ^{k-3}(\tau)\right. \\
& \left.+\left(\begin{array}{c}
k-3 \\
2
\end{array}\right) 2^{k-5} \cos ^{k-5}(\tau)-\left(\begin{array}{c}
k-4 \\
3
\end{array}\right) 2^{k-7} \cos ^{k-7}(\tau)+\cdots\right] .
\end{aligned}
$$

The approximate analytical solution is expressed explicitly in terms of $t$ by finally making the substitutions $\cos (\tau)=\operatorname{sech}(t \sqrt{\Omega})$ and $\sin (\tau)=\tanh (t \sqrt{\Omega})$. 


\section{Illustrations}

\subsection{Nonlinear wave problems}

We choose our first example from the study of nonlinear waves in physics. In this research subject the nonlinear Schrödinger equation, whose general form in one-dimensional space reads as [2, p. 155]

$$
i \frac{\partial u}{\partial z}+P \frac{\partial^{2} u}{\partial t^{2}}+h\left(|u|^{2}\right) u=0
$$

is one of the most encountered PDEs. In effect it is used to describe the slowly varying envelope of light electric fields propagating in optical Kerr media within the paraxial model of self-focusing, the evolution of wave-packets as, e.g., Langmuir waves in strong plasma turbulence [6]. It also arises in many other physical problems which include the evolution of water waves, the propagation of a heat pulse in a solid, the propagation of solitary waves in piezoelectric semiconductors, the study of nonlinear waves in a fluid-filled viscoelastic tube [10, p. 153].

By seeking the solution of Eq. (3.1) in the form $u(z, t)=\rho(t) e^{i \nu z}$ with $\nu, \rho(t) \in \mathbb{R}$ and assuming that $P \neq 0$, one obtains that $\rho(t)$ must be solution of the following ODE

$$
\ddot{\rho}-\frac{\nu}{P} \rho+\frac{1}{P} h\left(\rho^{2}\right) \rho=0 .
$$

Exact analytical solutions are obtainable in explicit form for Eq. (3.2) in the now classic case where $h$ is such that $h(\xi)=Q \xi$, and also for the cubic-quintic NLS equation for which $h(\xi)=Q \xi+Q_{2} \xi^{2}$, see [25]. For other forms of $h$ including polynomial functions of degree higher than two, no explicit analytical solution exist to our knowledge and it seems unavoidable to resort to approximation. We illustrate below the usefulness of our proposed method for such situations. However, for the sake of simplicity, we restrict ourselves to consider only monomial form of $h$. Specifically, we take $h(\xi)=\eta \xi^{3}$.

\subsubsection{Homoclinic orbit}

When $P, \nu$ and $\eta$ are all positive or all negative, Eq. (3.2) can be put in the form

$$
\ddot{\rho}-\omega^{2} \rho+\sigma^{2} \rho^{7}=0, \quad \omega>0, \quad \sigma>0 .
$$

The stationary solutions of this equation as well as their stability are easily determined to be $x_{+}^{(s)}=\sqrt[3]{\omega / \sigma}, x_{ \pm}^{(u)}=0$ and $x_{-}^{(s)}=-x_{+}^{(s)}$. It is also easy to verify that Eq. (3.3) above derives from the Hamiltonian

$$
H(\rho, \dot{\rho})=\frac{1}{2} \dot{\rho}^{2}-\frac{\omega^{2}}{2} \rho^{2}+\frac{\sigma^{2}}{8} \rho^{8} .
$$

The equation $H(A, 0)=H\left(x_{ \pm}^{(u)}, 0\right)$ has two solutions $A= \pm \sqrt[3]{2 \omega / \sigma}$ of which none is a stationary solution of Eq. (3.3). This indicates the existence of a pair homoclinic orbits that connect $x_{ \pm}^{(u)}=0$ to itself; and each of the $A$ is 
the abscissa of the turning point on one of these orbits. In order to determine approximate analytical expression for these solutions, Eq. (3.3) is first transformed into

$$
\frac{\Omega}{2}\left[(1+\cos (2 \tau)) \rho^{\prime \prime}-\rho^{\prime} \sin (2 \tau)\right]-\omega^{2} \rho+\sigma^{2} \rho^{7}=0
$$

using the change of variable in Eq. (2.5). Then, according to Eq. (2.8) the simplest approximation is taken as $\rho_{0}(\tau)=A \cos (\tau)$. The usual HB then enables us to obtain $\Omega_{0}=19 \omega^{2} / 8$. Thus the simplest approximate solution from our approach is given in explicit form as

$$
\rho_{0}(t)= \pm \sqrt[3]{2 \omega / \sigma} \operatorname{sech}(\sqrt{19 / 8} \omega t)
$$

The ansatz for the next approximation is obtained from Eq. (2.10) for $j=0$ and reads

$$
\rho_{1}(\tau)=A\left[\cos (\tau)+C_{1,3}(\cos (\tau)-\cos (3 \tau))\right] .
$$

We substitute this ansatz into Eq. (3.5) and put the ensuing equation in the form of a Fourier series. Then we solve the $\cos (\tau)$-coefficient of this series for $\Omega$. The solution is substituted in the $\cos (3 \tau)$-coefficient which is subsequently linearized with respect to, then solve for $C_{1,3}$. The expression obtained is simplified using $A= \pm \sqrt[3]{2 \omega / \sigma}$ and backward substituted in the expression of $\Omega$. Explicitly, we have

$$
\begin{aligned}
& C_{1,3}=-\frac{1}{22}, \quad \Omega_{1}=\frac{1644133001}{907039232} \omega^{2} \\
& \rho_{1}(t)= \pm \sqrt[3]{\frac{2 \omega}{\sigma}}\left[\frac{9}{11} \operatorname{sech}\left(t \sqrt{\Omega_{1}}\right)+\frac{2}{11} \operatorname{sech}^{3}\left(t \sqrt{\Omega_{1}}\right)\right] .
\end{aligned}
$$

We shall omit the details of further approximations by assuming that the procedure is now well established. Thus for brevity, the second and third order improved approximate solutions are given as follows

$$
\begin{aligned}
\Omega_{2} \approx & 1.570815495 \omega^{2}, \quad \rho_{2}(t) \approx \pm \sqrt[3]{\frac{2 \omega}{\sigma}}\left[0.8896999239 \operatorname{sech}\left(t \sqrt{\Omega_{2}}\right)\right. \\
& \left.-0.1747001803 \operatorname{sech}^{3}\left(t \sqrt{\Omega_{2}}\right)+0.2850002564 \operatorname{sech}^{5}\left(t \sqrt{\Omega_{2}}\right)\right], \\
\Omega_{3} \approx & 1.374552324 \omega^{2}, \quad \rho_{3}(t) \approx \pm \sqrt[3]{\frac{2 \omega}{\sigma}}\left[0.8141600989 \operatorname{sech}\left(t \sqrt{\Omega_{3}}\right)\right. \\
& -0.04102482745 \operatorname{sech}^{3}\left(t \sqrt{\Omega_{3}}\right)+0.09535597041 \operatorname{sech}^{5}\left(t \sqrt{\Omega_{3}}\right) \\
& \left.+0.1315087581 \operatorname{sech}^{7}\left(t \sqrt{\Omega_{3}}\right)\right] .
\end{aligned}
$$

It appears from equations Eq. (3.7), Eq. (3.9)-Eq. (3.11) that the various approximations are polynomials in the auxiliary function sech. The present approach offers a means for calculating the polynomial coefficients in an algorithmic way that can easily be implemented in an algebraic manipulator such as 


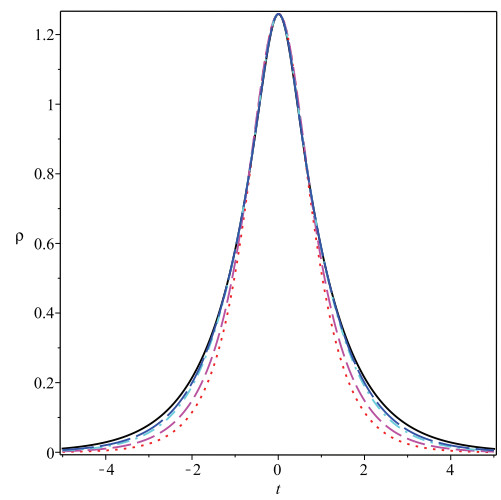

Figure 1. Comparison of various approximations of the homoclinic solution of Eq. (3.3) to the exact solution (solid black line) for $\omega=\sigma=1$. The red dotted, magenta dashed, cyan dash-dotted and blue long-dashed lines correspond to Eq. (3.6), Eqs. (3.8)-(3.9), Eqs. (3.10), and Eqs. (3.11) respectively.

Maple. It is worth notice that these coefficients are not decreasing monotonically as the power is increased.

The various approximations obtained above are plotted in Figure 1 together with the exact solution obtained numerically using the Runge-Kutta algorithm as implemented in the Maple package dsolve with options numeric and method=rk 45 . This figure shows that our approximations converge steadily toward the exact solution.

\subsubsection{Heteroclinic orbit}

Consider now the situation where $P \nu<0$ and $P \eta<0$. In this case, the signs of the $\omega^{2}$-term and $\sigma^{2}$-term of Eq. (3.3) are swapped, and similarly for Eq. (3.4). Then, the stability of the fixed points also changes so that $x_{+}^{(u)}=\sqrt[3]{\omega / \sigma}$, $x_{0}^{(s)}=0$ and $x_{-}^{(u)}=-x_{+}^{(u)}$. One can straightforwardly verify that the two unstable fixed points have the same energy value. Therefore, there exists an heteroclinic orbit connecting them. If approximate analytical expressions are needed for it, then our procedure provides the following for the zeroth through the third order improvement (details omitted for brevity):

$$
\begin{aligned}
\Omega_{0} & =\frac{29}{32}, \quad \rho_{0}(t)= \pm \sqrt[3]{\frac{\omega}{\sigma}} \tanh \left(\sqrt{\frac{29}{32}} \omega t\right) \\
\Omega_{1} & =\frac{315443280275}{235551657312}, \rho_{1}(t)= \pm \sqrt[3]{\frac{\omega}{\sigma}} \tanh \left(t \sqrt{\Omega_{1}}\right)\left[1-\frac{8}{27} \operatorname{sech}^{2}\left(t \sqrt{\Omega_{1}}\right)\right] \\
\Omega_{2} & \approx 1.365629149 \omega^{2}, \quad \rho_{2}(t) \approx \pm \sqrt[3]{\omega / \sigma} \tanh \left(t \sqrt{\Omega_{2}}\right) \\
& \times\left[1-0.3886044766 \operatorname{sech}^{2}\left(t \sqrt{\Omega_{2}}\right)+0.1347035012 \operatorname{sech}^{4}\left(t \sqrt{\Omega_{2}}\right)\right]
\end{aligned}
$$




$$
\begin{aligned}
\Omega_{3} \approx & 1.446122331 \omega^{2}, \quad \rho_{3}(t) \approx \pm \sqrt[3]{\omega / \sigma} \tanh \left(t \sqrt{\Omega_{3}}\right) \\
\times & {\left[1-0.4935148551 \operatorname{sech}^{2}\left(t \sqrt{\Omega_{3}}\right)+0.3170228201 \operatorname{sech}^{4}\left(t \sqrt{\Omega_{3}}\right)\right.} \\
& \left.-0.10474669950 \operatorname{sech}^{6}\right] .
\end{aligned}
$$

Figure 2 shows the comparison with the numerically obtained exact solution. Again very good accuracy is observed. In fact, for this particular case where the solution is antisymmetric, our approximate solution is indistinguishable from the exact one at the scale of the plot.

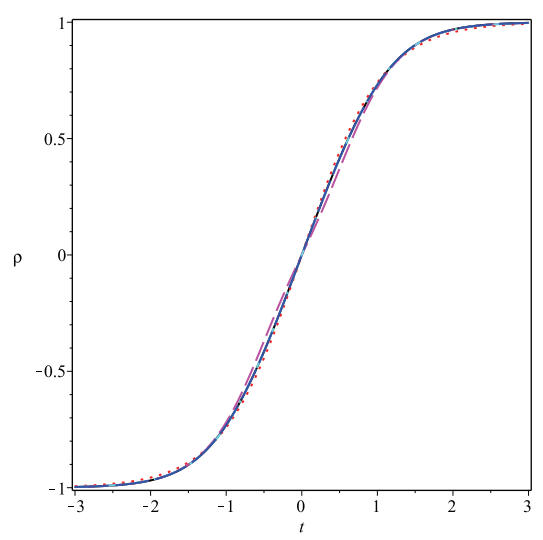

Figure 2. Comparison of various approximations of the heteroclinic solution to the exact solution (solid black line) for $\omega=\sigma=1$. The red dotted, magenta dashed, cyan dash-dotted and blue long-dashed lines correspond to Eq. (3.12), Eqs. (3.13), Eqs. (3.14), and Eq. (3.15) respectively. Only the first two approximates can be distinguished at the scale of the plot.

We leave this example with the observation that the asymptotic behavior of the system around a hyperbolic unstable equilibrium $x_{ \pm}^{(u)}$ is dictated by the eigenvalue of the linearized equation of motion at this point. We can then expect that the squared frequency-like parameter $\Omega$ should converge to this eigenvalue. This is in indeed the case, although the convergence may seem to be slow. In effect it decreases monotonically from $2.38 \omega^{2}$ toward the the squared eigenvalue $\omega^{2}$ calculated at the origin, for the homoclinic orbit. In the case of the heteroclinic orbit, it increases monotonically from $0.91 \omega^{2}$ to the squared eigenvalue $6 \omega^{2}$ which is obtained at $x_{ \pm}^{(u)}=\sqrt[3]{\omega / \sigma}$. Even though it may appear to be slow, the convergence is evidenced by the fact that the magnitude of the difference between the two values diminishes as more harmonic terms.

\subsection{Mechanical problem}

Our second example is taken from a mechanical problem. It consists of a simple pendulum subjected to linear feedback with desired periodic motions. The undamped autonomous version of the equation that governs the dynamics 
of this systems is given by [24]

$$
\ddot{x}+\sin (x)+\alpha x-\beta=0 .
$$

We choose $\beta=\alpha \pi$ and let $y=x-\pi$. Equation (3.16) then becomes

$$
\ddot{y}-\sin (y)+\alpha y=0,
$$

for which $y_{ \pm}^{(u)}=0$ is an hyperbolic saddle connected to itself by a pair of homoclinic orbits for $0<\alpha<1$. The amplitudes $A$ of these orbits are the solutions of the transcendental algebraic equation

$$
2 \cos (y)+\alpha y^{2}=0 .
$$

Applying the change of variable in Eq. (2.5) to Eq. (3.17) and using the identity $[1$, p. 361]

$$
\sin (\Gamma \cos (\theta))=2 \sum_{k=0}^{\infty}(-1)^{k} J_{2 k+1}(\Gamma) \cos ((2 k+1) \theta)
$$

we obtain the one-term approximation of the homoclinic orbits of Eq. (3.17) as

$$
\Omega_{0}=\frac{2\left(\alpha A-2 J_{1}(A)\right)}{A}, \quad y_{0}(t)=A \operatorname{sech}\left(t \sqrt{\Omega_{0}}\right) .
$$

Here and in what follows, $J_{m}$ designates the Bessel function of the first kind of order $m$ [1, p. 361].

An improved approximate solution can be considered in the form of (3.7). The exact computation of the Fourier coefficients necessary for our method is difficult for this expression due to the sine function. To manage this problem, these coefficients are calculated only after expanding the equation in powers of $C_{1,3}$. Then they can be obtained easily using the identity $[1$, p. 361$]$

$$
\cos (\Gamma \cos (\theta))=J_{0}(\Gamma)+2 \sum_{k=1}^{\infty}(-1)^{k} J_{2 k}(\Gamma) \cos (2 k \theta)
$$

in addition to Eq. (3.18). Retaining only terms that are linear with respect to $C_{1,3}$, the routine calculations described earlier lead to the following final form of the approximate solution:

$$
\begin{aligned}
& \Omega_{1}=\frac{2\left(\lambda_{1} C_{1,3}+\alpha A^{3}-2 A^{2} J_{1}(A)\right)}{\left(1-2 C_{1,3}\right) A^{3}} \\
& y_{1}(t)=A\left[\left(1+4 C_{1,3}\right) \operatorname{sech}\left(t \sqrt{\Omega_{1}}\right)-4 C_{1,3} \operatorname{sech}^{3}\left(t \sqrt{\Omega_{1}}\right),\right]
\end{aligned}
$$

where

$$
\begin{aligned}
& \lambda_{1}=\alpha A^{3}+8\left(6-A^{2}\right) J_{1}(A)-24 A J_{0}(A), \\
& C_{1,3}=\frac{\alpha A^{5}+8 J_{0}(A) A^{3}-16 J_{1}(A) A^{2}}{4 \alpha A^{5}-96\left(A^{2}-20\right) A J_{0}(A)-12\left(A^{4}-56 A^{2}+320\right) J_{1}(A)} .
\end{aligned}
$$


When the Fourier coefficients of either of the nonlinear functions $f(x(\tau))$ or $g(x(\tau))$ cannot be computed exactly in closed-form for a given non-harmonic truncation of $x(\tau)$ as in this example, a practical tricks consists of introducing a bookkeeping parameter $\mu$ and assuming that the $n^{\text {th }}$-order correction is or order $\mu^{n}$. In other world, Eq. (2.10) needs to be modified as

$$
x_{j+1}(\tau)=x_{j}(\tau)+\mu^{j+1} \sum_{i=1}^{j+1} C_{j+1,2 i+1}(\cos (\tau)-\cos ((2 i+1) \tau)),
$$

so that the functions may be expanded in power-series of $\mu$ which is set equal to 1 at thereafter. In this way the pseudo-frequency of the third approximate solution (or second order correction) for the controlled pendulum is found to be

$$
\Omega_{2}=\frac{2\left(\alpha A^{5}+\lambda_{2} C_{1,3}+\lambda_{2} C_{2,3}+\lambda_{3} C_{2,5}-2 A^{4} J_{1}(A)\right)}{\left(1-2 C_{1,3}-2 C_{2,3}+C_{2,5}\right) A^{5}},
$$

where

$$
\begin{aligned}
& C_{2,3}=\frac{2 \lambda_{6} \lambda_{7}-\lambda_{4} \lambda_{9}}{\lambda_{5} \lambda_{9}+2 \lambda_{6} \lambda_{8}}, \quad C_{2,5}=-\frac{2\left(\lambda_{4} \lambda_{8}+\lambda_{5} \lambda_{7}\right)}{\lambda_{5} \lambda_{9}+2 \lambda_{6} \lambda_{8}} \\
& \lambda_{2}=\alpha A^{5}-24 A^{3} J_{0}(A)-8 A^{2}\left(A^{2}-6\right) J_{1}(A), \\
& \lambda_{3}=\alpha A^{5}-24 A\left(7 A^{2}-80\right), J_{0}(A)-24\left(160-34 A^{2}+A^{4}\right) J_{1}(A) .
\end{aligned}
$$

The expressions of the other quantities $\lambda_{4}-\lambda_{9}$ which parameterize $C_{2,3}$ and $C_{2,5}$ are respectively given as

$$
\begin{aligned}
\lambda_{4} & =4 A^{7} \alpha C_{1,3}^{3}+12 A^{7} \alpha C_{1,3}^{2}+32 A^{6} J_{1}(A) C_{1,3}^{3}-9 A^{7} \alpha C_{1,3} \\
& -56 A^{6} J_{1}(A) C_{1,3}^{2}+480 A^{5} J_{0}(A) C_{1,3}^{3}+A^{7} \alpha+20 C_{1,3} J_{1}(A) A^{6} \\
& -448 A^{5} J_{0}(A) C_{1,3}^{2}-2880 A^{4} J_{1}(A) C_{1,3}^{3}+88 C_{1,3} J_{0}(A) A^{5} \\
& +2816 C_{1,3}^{2} J_{1}(A) A^{4}-7680 A^{3} J_{0}(A) C_{1,3}^{3}+8 J_{0}(A) A^{5} \\
& -656 C_{1,3} J_{1}(A) A^{4}+7680 A^{3} J_{0}(A) C_{1,3}^{2}+15360 A^{2} J_{1}(A) C_{1,3}^{3} \\
& +16 A^{2} C_{1,3}^{3} \lambda_{2}-16 J_{1}(A) A^{4}-1920 A^{3} J_{0}(A) C_{1,3}-15360 A^{2} J_{1}(A) C_{1,3}^{2} \\
& -10 A^{2} C_{1,3}^{2} \lambda_{2}+3840 C_{1,3} J_{1}(A) A^{2}+A^{2} C_{1,3} \lambda_{2}, \\
\lambda_{5} & =4 A^{7} \alpha C_{1,3}^{2}-4 A^{7} \alpha C_{1,3}+32 A^{6} J_{1}(A) C_{1,3}^{2}-5 A^{7} \alpha-32 C_{1,3} J_{1}(A) A^{6} \\
& +480 A^{5} J_{0}(A) C_{1,3}^{2}+20 J_{1}(A) A^{6}-480 C_{1,3} J_{0}(A) A^{5} \\
& -2880 C_{1,3}^{2} J_{1}(A) A^{4}+120 J_{0}(A) A^{5}+2880 C_{1,3} J_{1}(A) A^{4} \\
& -7680 A^{3} J_{0}(A) C_{1,3}^{2}-720 J_{1}(A) A^{4}+7680 A^{3} J_{0}(A) C_{1,3} \\
& +15360 A^{2} J_{1}(A) C_{1,3}^{2}+16 A^{2} C_{1,3}^{2} \lambda_{2}-1920 J_{0}(A) A^{3} \\
& -15360 C_{1,3} J_{1}(A) A^{2}-16 A^{2} C_{1,3} \lambda_{2}+3840 J_{1}(A) A^{2}+A^{2} \lambda_{2}, \\
\lambda_{6} & =96 A^{6} J_{1}(A) C_{1,3}^{2}-148 C_{1,3} J_{1}(A) A^{6}+1824 A^{5} J_{0}(A) C_{1,3}^{2} \\
& -1824 C_{1,3} J_{0}(A) A^{5}-19008 C_{1,3}^{2} J_{1}(A) A^{4}+19008 C_{1,3} J_{1}(A) A^{4}
\end{aligned}
$$


$-115200 A^{3} J_{0}(A) C_{1,3}^{2}+115200 A^{3} J_{0}(A) C_{1,3}+552960 A^{2} J_{1}(A) C_{1,3}^{2}$

- 552960 $C_{1,3} J_{1}(A) A^{2}-1290240 A J_{0}(A) C_{1,3}+1290240 C_{1,3}^{2} J_{0}(A) A$

$-645120 J_{1}(A)+26 A^{7} \alpha C_{1,3}+26 A^{2} C_{1,3}^{2} \lambda_{2}+16 A^{2} C_{1,3}^{2} \lambda_{3}$

$-10 A^{2} C_{1,3} \lambda_{2}-10 A^{2} C_{1,3} \lambda_{3}+322560 J_{0}(A) A+138240 J_{1}(A) A^{2}$

$-28800 J_{0}(A) A^{3}+456 J_{0}(A) A^{5}-4752 J_{1}(A) A^{4}+44 J_{1}(A) A^{6}$

$+2580480 J_{1}(A) C_{1,3}-2580480 C_{1,3}^{2} J_{1}(A)-10 A^{7} \alpha+A^{2} \lambda_{3}$, $\lambda_{7}=-7968 A^{6} J_{1}(A) C_{1,3}^{3}+7680 A^{6} J_{1}(A) C_{1,3}^{2}-53760 A^{5} J_{0}(A) C_{1,3}^{3}$

$-1704 C_{1,3} J_{1}(A) A^{6}+52992 A^{5} J_{0}(A) C_{1,3}^{2}+268800 A^{4} J_{1}(A) C_{1,3}^{3}$

$-12672 C_{1,3} J_{0}(A) A^{5}-267264 C_{1,3}^{2} J_{1}(A) A^{4}+645120 A^{3} J_{0}(A) C_{1,3}^{3}$

$+65664 C_{1,3} J_{1}(A) A^{4}-645120 A^{3} J_{0}(A) C_{1,3}^{2}-1290240 A^{2} J_{1}(A) C_{1,3}^{3}$

$+161280 A^{3} J_{0}(A) C_{1,3}+1290240 A^{2} J_{1}(A) C_{1,3}^{2}-322560 C_{1,3} J_{1}(A) A^{2}$

$+108 C_{1,3} J_{0}(A) A^{7}+6 C_{1,3} J_{1}(A) A^{8}-24 A^{8} J_{1}(A) C_{1,3}^{2}$

$-576 A^{7} J_{0}(A) C_{1,3}^{2}+16 A^{8} J_{1}(A) C_{1,3}^{3}+624 A^{7} J_{0}(A) C_{1,3}^{3}$

$-192 J_{0}(A) A^{5}+384 J_{1}(A) A^{4}-72 J_{1}(A) A^{6}+J_{1}(A) A^{8}+12 J_{0}(A) A^{7}$

$+6 A^{9} \alpha C_{1,3}^{2}-3 A^{9} \alpha C_{1,3}-3 A^{4} C_{1,3}^{2} \lambda_{2}+6 A^{4} C_{1,3}^{3} \lambda_{2}$,

$\lambda_{8}=-16 A^{8} J_{1}(A) C_{1,3}^{2}+3 \alpha A^{9}+16 C_{1,3} J_{1}(A) A^{8}-624 A^{7} J_{0}(A) C_{1,3}^{2}$

$-10 J_{1}(A) A^{8}+624 C_{1,3} J_{0}(A) A^{7}+7968 A^{6} J_{1}(A) C_{1,3}^{2}-156 J_{0}(A) A^{7}$

$-7968 C_{1,3} J_{1}(A) A^{6}+53760 A^{5} J_{0}(A) C_{1,3}^{2}+1992 J_{1}(A) A^{6}$

$-53760 C_{1,3} J_{0}(A) A^{5}-268800 C_{1,3}^{2} J_{1}(A) A^{4}-6 A^{4} C_{1,3}^{2} \lambda_{2}$

$+13440 J_{0}(A) A^{5}+268800 C_{1,3} J_{1}(A) A^{4}+6 A^{4} C_{1,3} \lambda_{2}$

- $645120 A^{3} J_{0}(A) C_{1,3}^{2}-67200 J_{1}(A) A^{4}+645120 A^{3} J_{0}(A) C_{1,3}$

$+1290240 A^{2} J_{1}(A) C_{1,3}^{2}-161280 J_{0}(A) A^{3}-1290240 C_{1,3} J_{1}(A) A^{2}$

$+322560 J_{1}(A) A^{2}$,

$\lambda_{9}=-73536 A^{6} J_{1}(A) C_{1,3}^{2}+73536 C_{1,3} J_{1}(A) A^{6}-798720 A^{5} J_{0}(A) C_{1,3}^{2}$

$+798720 C_{1,3} J_{0}(A) A^{5}+6758400 C_{1,3}^{2} J_{1}(A) A^{4}-6758400 C_{1,3} J_{1}(A) A^{4}$

$+36126720 A^{3} J_{0}(A) C_{1,3}^{2}-36126720 A^{3} J_{0}(A) C_{1,3}$

$-165150720 A^{2} J_{1}(A) C_{1,3}^{2}+165150720 C_{1,3} J_{1}(A) A^{2}$

$+371589120 A J_{0}(A) C_{1,3}-371589120 C_{1,3}^{2} J_{0}(A) A-4128 C_{1,3} J_{0}(A) A^{7}$

$-208 C_{1,3} J_{1}(A) A^{8}+96 A^{8} J_{1}(A) C_{1,3}^{2}+4128 A^{7} J_{0}(A) C_{1,3}^{2}$

$+185794560 J_{1}(A)-92897280 J_{0}(A) A-41287680 J_{1}(A) A^{2}$

$+9031680 J_{0}(A) A^{3}-199680 J_{0}(A) A^{5}+1689600 J_{1}(A) A^{4}$

$-18384 J_{1}(A) A^{6}-743178240 J_{1}(A) C_{1,3}+743178240 C_{1,3}^{2} J_{1}(A)$

$+74 J_{1}(A) A^{8}+1032 J_{0}(A) A^{7}-24 \alpha A^{9}+4 A^{9} \alpha C_{1,3}^{2}+52 A^{9} \alpha C_{1,3}$

$+56 A^{4} C_{1,3}^{2} \lambda_{2}+12 A^{4} C_{1,3}^{2} \lambda_{3}-25 A^{4} C_{1,3} \lambda_{2}-6 A^{4} C_{1,3} \lambda_{3}$. 
Figure 3 show the comparison of the three approximations to the exact solution obtained via numerical integration. The third approximate solution appears to superimpose almost perfectly to the the exact solution; while the from the first two a steady convergence is observed once again.

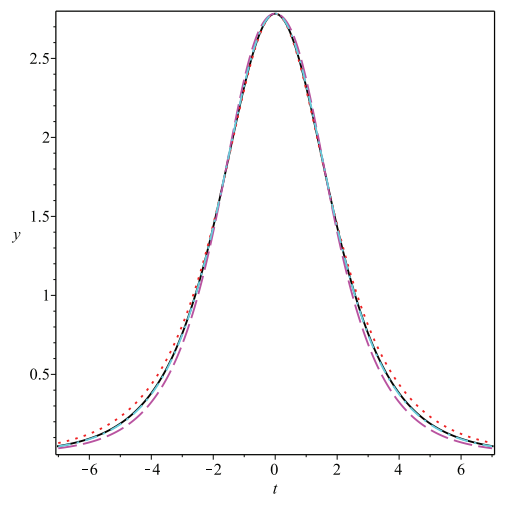

Figure 3. Comparison of various approximations of the homoclinic solution of Eq. (3.3) to the exact solution (solid black line) for $\omega=\sigma=1$. The red dotted, magenta dashed, cyan dash-dotted and blue long-dashed lines correspond to Eq. (3.6), Eqs. (3.8)-(3.9), Eqs. (3.10), and Eqs. (3.11) respectively.

\subsection{An example of asymmetric problem}

In Section 2, only second order ODEs that have the odd-parity property were considered in the outline of our proposed technique because they allow for a clear and straightforward formulation. We aim now to demonstrate through an example that our method is also applicable beyond those second order symmetric problems. To this end, we consider a perturbed KdV equation [16]

$$
u_{\tau}+6 u u_{x}+u_{x x x}+\varepsilon\left(\alpha u_{x x x x x x}+\beta u u_{x x x}+3 \lambda u_{x} u_{x x}+\sigma u^{2} u_{x}\right)=0,
$$

where a subscript variable means derivation with respect to this variable. Looking for traveling wave solutions with constant profile and velocity, we let $t=x-\eta \tau$. This transforms equation Eq. (3.22) into a sixth order ODE that we integrate once to obtain

$$
\ddot{u}-\eta u+3 u^{2}+\varepsilon\left(\alpha \frac{\mathrm{d}^{5} u}{\mathrm{~d} t^{5}}+\beta u \ddot{u}+\frac{3 \lambda-\beta}{2}(\dot{u})^{2}+\frac{\sigma}{3} u^{3}\right)=0,
$$

taking the constant of integration equal to zero. One can realize that the fifth order derivative in the last equation above is a damping-like term. It will allow for soliton solution with constant parameters (amplitude, width and velocity) being investigated only when very delicate relationships exist between these parameters. The application of our method to this case will be considered 
elsewhere. For now, we drop this term here onward by setting $\alpha=0$. Then equation (3.23) can obviously be put in the form Eq. (2.1) but with functions $g$ and $f$ not odd. After applying the key change of independent variable Eq. (2.5) to Eq. (3.23), we seek an initial approximation to the solution of the resulting parametric ODE in the form

$$
u_{0}(t)=A(1+\cos (2 t))
$$

Upon substituting this ansatz into the equation, it takes the form

$$
E_{0}+E_{2} \cos (2 t)+E_{4} \cos (4 t)+E_{6} \cos (6 t)=0 .
$$

We form a system of two linear algebraic equations in $\Omega$ and $\eta$ by equating $E_{0}$ and $E_{2}$ to zero. The solution of this system is easily found to be

$$
\begin{aligned}
& \Omega_{0}=\frac{(18+5 \varepsilon \sigma A) A}{9[2+(2 \beta+\lambda) \varepsilon A]}, \quad \eta_{0}=\frac{A \mathcal{N}}{9[2+(2 \beta+\lambda) \varepsilon A]}, \\
& \mathcal{N}=144+10 \sigma(3 \lambda+\beta) \varepsilon^{2} A^{2}+(25 \sigma+135 \lambda+90 \beta) \varepsilon A,
\end{aligned}
$$

which yields

$$
u_{0}(x, \tau)=2 A \operatorname{sech}^{2}\left(\left(x-\eta_{0} \tau\right) \sqrt{\Omega_{0}}\right)
$$

as the first approximate solution to the perturbed $\mathrm{KdV}$ equation (3.22) with $\alpha=0$.

Proceeding further necessitates to coin an appropriate increment and add it to the right hand side of Eq. (3.24). In this regard we follow the simple rule suggested in [27]. It recommends that the harmonics to include in the increment to the ansatz of a given stage should be higher than or equal to the least harmonic of the residual terms of that stage. Combining this rule with the requirement $u_{1}(0)=u_{0}(0)$ and the boundary conditions Eq. (2.7), the ansatz of the next stage of approximation for our current example is taken as

$$
u_{1}(t)=A[1+\cos (2 t)+b(1-\cos (4 t))],
$$

the corresponding explicit form of which is

$$
u_{1}(x, \tau)=2 A(1+4 b) \operatorname{sech}^{2}\left(\left(x-\eta_{1} \tau\right) \sqrt{\Omega_{1}}\right)-8 A b \operatorname{sech}^{4}\left(\left(x-\eta_{1} \tau\right) \sqrt{\Omega_{1}}\right) .
$$

In these equations $b, \Omega_{1}$ and $\eta_{1}$ are to be determined. Once again, the routine HB procedure using Eq. (3.26) yields a Fourier series similar to Eq. (3.25) where the bias term and the coefficient of the $\cos (2 t)$-term are also linear in $\Omega$ and $\eta$. Solving these coefficients for the latter gives

$$
\Omega_{1}=\frac{4 A \chi_{1}}{\Delta}, \quad \eta_{1}=\frac{A \chi_{2}}{\Delta}
$$


with

$$
\begin{aligned}
\Delta & =72-48 b-528 \varepsilon \beta A b^{2}-84 \varepsilon \beta A b+288 A \varepsilon \lambda b^{2}+108 A \varepsilon \lambda b \\
& -144 b^{2}-288 A \varepsilon \lambda b^{3}+144 \varepsilon \beta A b^{3}+36 A \varepsilon \lambda+72 \varepsilon \beta A \\
\chi_{1} & =18+36 b+5 \varepsilon \sigma A-18 b^{2}+12 \varepsilon \sigma A b-4 \varepsilon \sigma A b^{3} \\
\chi_{2} & =288-144 b+648 \varepsilon \beta A b^{4}+120 \varepsilon^{2} \beta A^{2} b^{5} \sigma-41 \varepsilon^{2} \beta A^{2} \sigma b \\
& -248 \varepsilon^{2} \beta A^{2} b^{4} \sigma-384 \varepsilon^{2} \beta A^{2} b^{2} \sigma-478 \varepsilon^{2} \beta A^{2} b^{3} \sigma+60 A^{2} \varepsilon^{2} \lambda \sigma \\
& +20 \varepsilon^{2} \beta A^{2} \sigma+258 A^{2} \varepsilon^{2} \lambda b^{3} \sigma-240 A^{2} \varepsilon^{2} \lambda b^{5} \sigma+87 A^{2} \varepsilon^{2} \lambda b \sigma \\
& -48 A^{2} \varepsilon^{2} \lambda b^{4} \sigma+318 A^{2} \varepsilon^{2} \lambda b^{2} \sigma-48 \varepsilon \sigma A b^{2}-120 \varepsilon \sigma A b^{4}-1296 A \varepsilon \lambda b^{4} \\
& -2232 \varepsilon \beta A b^{2}-216 \varepsilon \beta A b+1566 A \varepsilon \lambda b^{2}+432 A \varepsilon \lambda b+50 \varepsilon \sigma A \\
& -432 b^{2}-648 b^{3}-24 \varepsilon \sigma A b-136 \varepsilon \sigma A b^{3}+432 A \varepsilon \lambda b^{3}-1728 \varepsilon \beta A b^{3} \\
& +270 A \varepsilon \lambda+180 \varepsilon \beta A .
\end{aligned}
$$

To complete the determination of the approximation, equations (3.27)-(3.28c) are substituted in the coefficient of the cos $(4 t)$-term, which is subsequently linearized with respect to $b$. From the ensuing linear equation, $b$ reads as

$$
b=\frac{\varepsilon A[2 \sigma(\beta+3 \lambda) \varepsilon A+27 \lambda+18 \beta-3 \sigma]}{24+\sigma(5 \beta+\lambda) \varepsilon^{2} A^{2}+7(3 \beta+\sigma) \varepsilon A} .
$$

We can observe that the dependency of $b$ on the parameters of Eq. (3.22) is such that $b$ vanishes when the perturbation therein is switched off (i.e., $\varepsilon=0$ ), as expected. Being rational in these parameters, the accuracy of this expression is however not limited to finitely small values of $\varepsilon$, in contrast to results that would be obtained using classical perturbation methods.

\section{Conclusions}

In this paper, we have proposed a technique for obtaining analytical approximations to homoclinic and heteroclinic solutions of some ODEs. It consists essentially of the well-known method of harmonic balance; but which is applied only after effecting a simple and explicit change of the independent variable that we have indicated. From the examples used to illustrate the proposed approach, it appears to produce accurate explicit approximations which are steadily converging to the exact solutions with increased level of approximations. The proposed method is also very easy and straightforward to use and can be easily implemented in computer algebra systems.

Our examples included both symmetric and asymmetric ODEs, but was limited to second order conservative systems. However, since the proposed method leans on the HB which is known to be applicable to ODEs of any order [19], ODEs of arbitrary order can also certainly be investigated using the proposed method. A path of further widening this method currently being investigated is its applicability to damped systems. 


\section{References}

[1] M. Abramowitz and I.A. Stegun. Handbook of Mathematical Functions with Formulas, Graphs, and Mathematical Tables. Dover, New York, 1972.

[2] G.P. Agrawal. Nonlinear Fiber Optics. Academic Press, Oxford, 2013.

[3] P. Amore and A. Aranda. Improved Lindstedt-Poincaré method for the solution of nonlinear problems. J. Sound Vib., 283(3-5):1115-1136, 2005. https://doi.org/10.1016/j.jsv.2004.06.009.

[4] J. Awrejcewicz, I.V. Andrianov and L.I. Manevitch. Asymptotic Approaches in Nonlinear Dynamics: New trends and applications. Springer, Berlin, 1998. https://doi.org/10.1007/978-3-642-72079-6.

[5] A. Beléndez, E. Gimeno, M.L. Álvarez, D.I. Méndez and A. Hernández. Application of a modified rational harmonic balance method for a class of strongly nonlinear oscillators. Phys. Lett. A, 372(39):6047-6052, 2008. https://doi.org/10.1016/j.physleta.2008.08.024.

[6] L. Bergé and A. Couairon. A variational method for extended nonlinear Schrödinger systems. Physica D, 152-153(9):752-762, 2001. https://doi.org/10.1016/S0167-2789(01)00208-1.

[7] S.H. Chen, Y.Y. Chen and K.Y. Sze. A hyperbolic perturbation method for determining homoclinic solution of certain strongly nonlinear autonomous oscillators. J. Sound Vib., $\mathbf{3 2 2 ( 1 - 2 ) : 3 8 1 - 3 9 2 , ~} 2009$. https://doi.org/10.1016/j.jsv.2008.11.015.

[8] S.H. Chen, Y.Y. Chen and K.Y. Sze. Homoclinic and heteroclinic solutions of cubic strongly nonlinear autonomous oscillators by hyperbolic Lindstedt-Poincaré method. Sci. China Tech. Sci., 53(3):692-702, 2010. https://doi.org/10.1007/s11431-010-0069-5.

[9] Y.Y. Chen, L.W. Yang, K.Y. Sze and S.H. Chen. Generalized hyperbolic perturbation method for homoclinic solutions of strongly nonlinear autonomous systems. Appl. Math. Mech. -Engl. Ed., 39:1137-1152, 2012.

[10] L. Debnath. Nonlinear Partial Differential Equations for Scientists and Engineers. Springer, New York, 2012. https://doi.org/10.1007/978-0-8176-8265-1.

[11] J.J. Feng, Q.C. Zhang and W. Wang. The construction of homoclinic and heteroclinic orbitals in asymmetric strongly nonlinear systems based on the Padé approximant. Chin. Phys. B, 20(9):090202, 2011. https://doi.org/10.1088/16741056/20/9/090202.

[12] J.J. Feng, Q.C. Zhang and W. Wang. A novel construction of homoclinic and heteroclinic orbits in nonlinear oscillators by a perturbation-incremental method. Nonlinear Dyn., 64(3):221-236, 2011. https://doi.org/10.1007/s11071-011-99909.

[13] I.S. Gradshteyn and I.M. Ryzhik. Table of Integrals, Series, and Products. Academic Press, San Diego, seventh edition, 2007.

[14] J.-H. He. Modified Lindstedt-Poincare methods for some strongly non-linear oscillations, part i: expansion of a constant. Int. J. Non-Linear Mech., 37(2):309314, 2002. https://doi.org/10.1016/S0020-7462(00)00116-5.

[15] D.W. Jordan and P. Smith. Nonlinear Ordinary Differential Equations: Problems and Solutions: A Sourcebook for Scientists and Engineers. Oxford Texts in Applied \& Engineering Mathematics. Oxford University Press, USA, 2007. ISBN $9780199212033,0199212031$. 
[16] Y. Kai. Generalized hyperbolic perturbation method for homoclinic solutions of strongly nonlinear autonomous systems. Phys. Scr., 91(2):025202, 2016. https://doi.org/10.1088/0031-8949/91/2/025202.

[17] C.W. Lim and B.S. Wu. A new analytical approach to the Duffing-harmonic oscillator. Phys. Lett. A, 311(4-5):365-373, 2003. https://doi.org/10.1016/S03759601(03)00513-9.

[18] C.W. Lim, B.S. Wu and W.P. Sun. Higher accuracy analytical approximations to the Duffing-Harmonic oscillator. J. Sound Vib., 296(4-5):1039-1045, 2006. https://doi.org/10.1016/j.jsv.2006.02.020.

[19] R.E. Mickens. Comments on the method of harmonic balance. J. Sound Vib., 94:456-460, 1984. https://doi.org/10.1016/S0022-460X(84)80025-5.

[20] R.E. Mickens. Fourier representations for periodic solutions of odd-parity systems. J. Sound Vib., 258(2):398-401, 2002. https://doi.org/doi:10.1006/jsvi.5200.

[21] A.H. Nayfeh. Introduction to Perturbation Techniques. Wiley, New York, 1981.

[22] J.A. Sanders, F. Verhulst and J. Murdock. Averaging Methods in Nonlinear Dynamical Systems. Springer, New-York, 2007.

[23] B.S. Wu, W.P. Sun and C.W. Lim. An analytical approximate technique for a class of strongly non-linear oscillators. Int. J. Non-Linear Mech., 41(6-7):766774, 2006. https://doi.org/10.1016/j.ijnonlinmec.2006.01.006.

[24] K. Yagasaki. A simple feedback control system: bifurcation of periodic orbits and chaos. Nonlinear Dyn., 9:391-417, 1996. https://doi.org/10.1007/BF01833363.

[25] S.B. Yamgoué and J.H. Kamga. Analytical solutions of undamped and autonomous cubic-quintic duffing equation. American Journal of Physics and Applications, 3:159-165, 2015. https://doi.org/10.11648/j.ajpa.20150305.11.

[26] S.B. Yamgoué and T.C. Kofané. On the analytical approximation of damped oscillations of autonomous single degree of freedom oscillators. Int. J. Non-Linear Mech., 41(10):1248-1254, 2006. https://doi.org/10.1016/j.ijnonlinmec.2007.01.002.

[27] S.B. Yamgoué, B. Nana and O.T. Lekeufack. Improvement of harmonic balance using Jacobian elliptic functions. Journal of Applied Mathematics and Physics, 3:680-690, 2015. https://doi.org/10.4236/jamp.2015.36081.

[28] Serge Bruno Yamgoué, Jean Roger Bogning, Aurélien Kenfack Jiotsa and Timoléon Crépin Kofané. Rational harmonic balance-based approximate solutions to nonlinear single-degree-of-freedom oscillator equations. Phys. Scr., 81(3), 2010. https://doi.org/10.1088/0031-8949/81/03/035003. Available from Internet: stacks .iop.org/PhysScr/81/035003.

[29] Serge Bruno Yamgoué and Timoléon Crépin Kofané. Linearized harmonic balance based derivation of slow flow for some class of autonomous single degree of freedom oscillators. Int. J. Non-Linear Mech., 43(9):993-999, 2008. ISSN 0020-7462. https://doi.org/10.1016/j.ijnonlinmec.2008.05.001. 